\title{
Hepatic Antifibrotic Pharmacotherapy: Are We Approaching Success?
}

\author{
Yue Chang ${ }^{1,2}$ and Hai Li*1,2 \\ ${ }^{1}$ Division of Gastroenterology and Hepatology, Tianjin Xiqing Hospital, Tianjin, China; ${ }^{2}$ Tianjin Key Laboratory of \\ Hepatopancreatic Fibrosis and Molecular Diagnosis and Treatment, Tianjin, China
}

\begin{abstract}
The incidence rate and mortality of liver fibrosis caused by various etiologies are high throughout the world. Liver fibrosis, the subsequent cirrhosis and other serious related complications threaten the health of patients and represent a serious medical burden; yet, there is still a lack of approved methods to prevent or reverse liver fibrosis. Therefore, effective hepatic antifibrotic drugs are urgently needed. The activation and proliferation of hepatic stellate cells are still the mechanisms of fibrosis that remain the focus of therapeutic research. In recent years, significant progress has been made in the development and applicability of antifibrosis drugs. In this review, we summarize the effectiveness and safety of available antifibrosis drugs utilizing different targets. In addition, some characteristics of antifibrosis drugs in phase II and III trials are introduced in detail.
\end{abstract}

Citation of this article: Chang Y, Li H. Hepatic antifibrotic pharmacotherapy: Are we approaching success? J Clin Transl Hepatol 2020;8(2):222-229. doi: 10.14218/JCTH.2020.00026.

\section{Introduction}

Liver cells usually regenerate after injury, but when injury and inflammation persist, the liver cannot regenerate normally and fibrosis will occur. Liver fibrosis is a pathological outcome of the repair response to chronic liver injury caused by any etiology, such as hepatitis B or C virus infection (HBV/HCV), nonalcoholic fatty liver disease (NAFLD), alcoholic steatohepatitis, autoimmune hepatitis, or cholestatic liver disease. Tissue remodeling and repair can lead to the production and deposition of a large number of collagens, fibronectin, undulin, laminin, and other extracellular matrixes (ECMs) and eventually to the formation of scar tissue. ${ }^{1}$ Long-term liver fibrosis will promote the accumulation of a fibrous

Keywords: Antifibrotic agents; Fibrosis; Liver; Reversal; Pharmacotherapy. Abbreviations: $A E$, adverse event; $A S K 1$, apoptosis signal-regulating kinase 1 ; CB1, cannabinoid receptor $1 ;$ CCR, CC chemokine receptor; ECM, extracellular matrix; FGF, fibroblast growth factor; FXR, farnesoid $X$ receptor; GLP-1, glucagon-like peptide-1; HBV/HCV, hepatitis B or C virus infection; HSC, hepatic stellate cell; HSP-47, heat shock protein-47; LOXL2, lysyl oxidase-like protein 2; MF, myofibroblast; MMP, matrix metalloproteinase; NA, nucleos(t)ide analog; NAFLD, nonalcoholic fatty liver disease; NASH, nonalcoholic steatohepatitis; NF-кB, nuclear factor-кB; NK, natural killer; OCA, obeticholic acid; PDGF, platelet-derived growth factor; PPAR, peroxisome proliferator-activated receptor; TGF, transforming growth factor; TIMP, tissue inhibitor of metalloproteinase.

Received: 6 April 2020; Revised: 10 May 2020; Accepted: 1 June 2020

*Correspondence to: Hai Li, Division of Gastroenterology and Hepatology, Tianjin Xiqing Hospital, No. 403 Xiqing Road, Xiqing District, Tianjin 300380, China. Tel: +86-22-60578765, Fax: +86-22-24370605, E-mail: haili_tj@sina.com matrix and destroy the normal function and structure of the liver. If left untreated, it will eventually progress to liver cirrhosis or carcinoma, which are the major causes of death due to chronic liver disease. Therefore, there is a dire need for an antifibrotic drug that can not only inhibit the progression of hepatic fibrosis but also reverse its progression.

However, to date, there is no effective chemical drug in the clinic for the treatment of liver fibrosis. Therefore, research on hepatic antifibrotic drugs is a 'hot topic'. At present, the main drug treatment strategies for fibrosis include the treatment of primary diseases, control of the inflammation, regulation of ECM synthesis and degradation, improvement in liver parenchyma cell injury, and apoptosis. Although there are no approved pharmacotherapies for fibrosis, sustained effort and remarkable progress have been made in the research on antifibrosis drugs in recent years, particularly for drugs for NAFLD-related fibrosis. The present review will emphasize the progress that has been made in efficacy and safety of potential drugs for the treatment of fibrosis and highlight underlying challenges in the future.

\section{Activated hepatic stellate cells (HSCs) are still the primary effector cell of fibrosis}

Myofibroblasts (MFs) are the main cells that produce ECM (e.g., collagens) in the process of chronic liver cell damage. MFs do not exist in normal liver tissue. The major source of MFs is HSCs, although a small part of MFs comes from portal vein fibroblasts, ${ }^{2}$ hematopoietic stem cell fibroblasts, and bone marrow-derived fibrocytes. ${ }^{3}$ Interestingly, in the model of cholestatic liver injury, portal vein fibroblasts are the major source of MFs at the onset of injury, but HSCs are still the main source of MFs in the later stages. ${ }^{4}$ Nevertheless, it is controversial whether MFs originate from hepatocytes or cholangiocytes by the epithelial-to-mesenchymal transition or endothelial mesenchymal transition. ${ }^{5}$

In the healthy liver, HSCs show a quiescent phenotype. HSCs are located in the space of Disse, accounting for $5-8 \%$ of the total cells of the liver. ${ }^{6}$ There is much evidence that the activation of HSCs plays a critical role in fibrosis. Transforming growth factor (TGF)- $\beta$, osteopontin, and platelet-derived growth factor (PDGF) are the most important cytokines that promote the activation of HSCs and the proliferation of ECM. Many other cytokines and intracellular signal transduction pathways are also involved in the activation of HSCs. Therefore, drugs targeting the activation of HSCs will become a therapeutic strategy for hepatic antifibrosis.

Reducing the number of activated HSCs is essential for reversing and treating liver fibrosis. The three main pathways 
that can help eliminate activated HSCs are the return to quiescent phenotype, apoptosis, and senescence (Fig. 1). ${ }^{7-9}$ At present, there is solid evidence that the reversal of HSC activation to the quiescent cell state plays a dominant role. ${ }^{10}$ Thus, promoting the apoptosis of HSCs may be a potential antifibrotic target. In addition, multiple other cell types and factors play important roles in the process of liver fibrosis, such as immune cells, particularly macrophages, ${ }^{11}$ liver progenitor cells, autophagy, ${ }^{12}$ and epigenetics. ${ }^{13,14}$ Pathways and signals derived from intrahepatic or extrahepatic events also provide some potential targets for the drug treatment of liver fibrosis.

\section{Pharmacological therapy strategies for liver fibrosis}

Currently, with a better understanding of the pathogenesis of fibrosis, an increasing number of potential drugs that reverse fibrosis are in phase II or III trials. Here, we briefly review the current status of promising antifibrotic drugs in clinical trials (Table 1). The following represent the latest advances in pharmacological therapy strategies for antifibrosis and are outlined in Fig. 2.

\section{Curing or controlling the primary disease}

There is no doubt that the control or cure of primary liver disease is an efficient and effective way to reverse the progression of fibrosis. Many studies have proven that if the underlying etiology is effectively controlled or eliminated, liver fibrosis can be reversed, the structure and function of the liver can be restored to normal, and the risk of developing cirrhosis and tumors can be decreased. ${ }^{15}$

The most complete clinical evidence comes from chronic viral hepatitis. Clearance of HCV or long-term effective inhibition of HBV with potent nucleos(t)ide analogs (NAs) can effectively reduce and even reverse the progression of fibrosis and cirrhosis. ${ }^{16,17}$ It is worth noting that if there has been liver cirrhosis with significant portal hypertension, even after virologic cure, there may still be signs of clinical disease progression in a short period of time, including recurrent complications. In nonalcoholic steatohepatitis (NASH), a loss of up to $10 \%$ of total body weight can improve the fibrosis stage. ${ }^{18}$ It was observed that, despite sustained virologic response, $8-12 \%$ of patients with HCV still showed progress

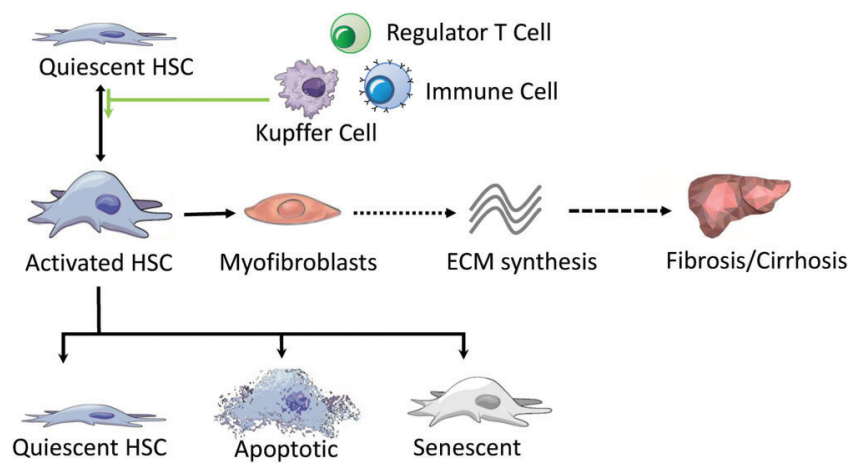

Fig. 1. Pathogenesis of liver fibrosis.

The schematic summarizes the fate of hepatic stellate cells and their role in liver fibrosis.

Abbreviations: HSC, hepatic stellate cell; ECM, extracellular matrix.

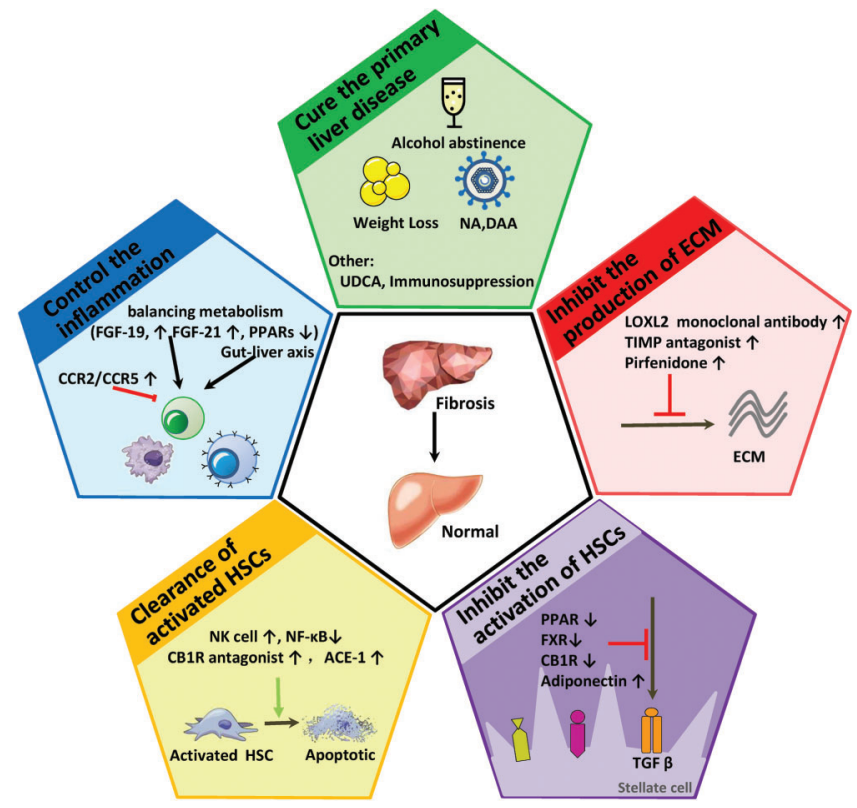

Fig. 2. Pharmacological therapy strategies for hepatic antifibrosis.

Abbreviations: ACE, angiotensin converting enzyme inhibitors; $\mathrm{CB} 1$, cannabinoid receptor 1 ; CCR, CC chemokine receptor; DAA, directacting antiviral agents; ECM, extracellular matrix; FGF, fibroblast growth factor; FXR, farnesoid $X$ receptor; HSC, hepatic stellate cell; $\mathrm{NA}$, nucleos(t)ide analog; NK, natural killer; PPARs, peroxisome proliferator-activated receptor; TGF, transforming growth factor; LOXL2, lysyl oxidase-like protein 2; TIMP, tissue inhibitor of metalloproteinase.

in the degree of fibrosis or cirrhosis ${ }^{17,19}$ and still retained a $5 \%$ risk of liver cancer. ${ }^{20}$ The possible cause of fibrosis progression or liver primary cancer is that antiviral therapy starts too late and is more likely to be associated with other underlying liver diseases, most of which are NAFLDs.

In recent years, liver fibrosis and cirrhosis caused by NAFLD have attracted increasing attention, as these will become the major etiologies of liver transplantation or hepatocellular carcinoma in the near future. ${ }^{21}$ Insulin resistance, oxidative stress, and metabolic disorders are the main pathological bases for the occurrence of NAFLD and the progression of fibrosis. Many therapeutic strategies and new research drugs for NAFLD fibrosis mainly target reducing insulin resistance or abnormal metabolism to reduce the production of free fatty acids, lipotoxicity, excessive accumulation of triglycerides in hepatocytes, mitochondrial dysfunction, and endoplasmic reticulum stress. ${ }^{22}$ Many agonists of receptors for the NAFLD metabolic pathway have been found to be effective in inhibiting fibrosis, such as farnesoid $X$ receptor (FXR) antagonist, peroxisome proliferator-activated receptors (PPARs), and glucagon-like peptide-1 (GLP-1). On the one hand, FXR plays a central role in glucose and lipid metabolism. On the other hand, FXR can also down-regulate the adipogenesis inducer SREPB-1c to induce fibroblast growth factor (FGF) 19 and reduce the production of endogenous bile acids. ${ }^{23}$ Obeticholic acid (OCA), a strong FXR agonist, has been demonstrated to improve biomarkers of inflammation and reduce the degree of fibrosis stage in patients with type 2 diabetes and NAFLD. ${ }^{24,25}$ Of course, other nonsteroidal FXR ligands, including AKN-083 (Allergan, 
Chang Y. et al: Pharmacotherapy for hepatic anti-fibrotic

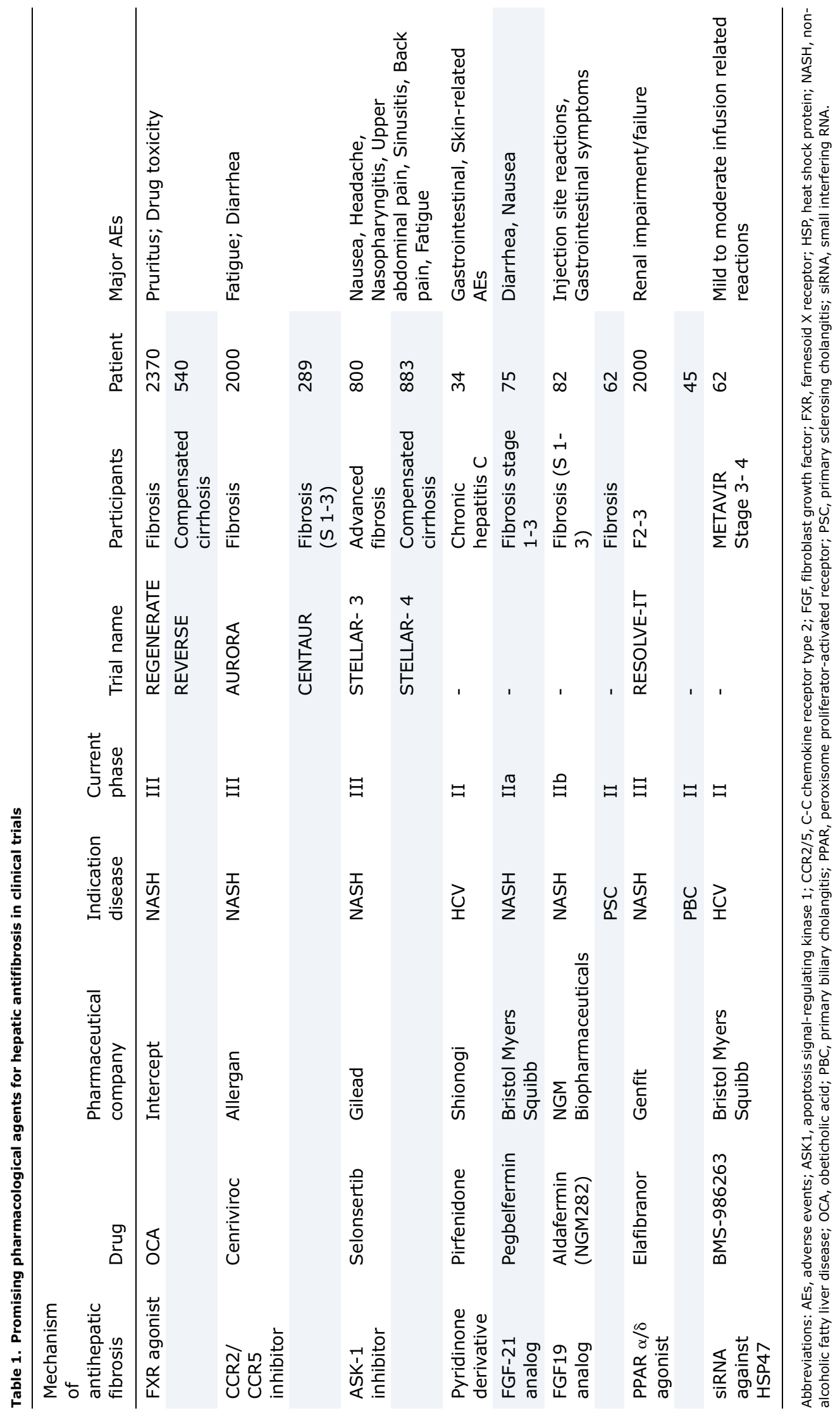


Dublin, Ireland), troifexor (Novartis, Basel, Switzerland), LMB763 (Novartis), and GS-9674 (Gilead Sciences, Foster City, CA, USA), are also in vigorous experiments and studies, and are expected to become prospects for antifibrosis drugs. In addition to the FXR agonist, PPARs (PPAR $\alpha$, PPAR $\beta$ / $\delta$, and PPAR $\gamma$ ) have been widely tested in NAFLD. Although pioglitazone (PPAR $\gamma$ agonist) has been found to reduce liver fibrosis in patients with NASH without type 2 diabetes, ${ }^{26}$ the limitations of patients with heart failure and drug-related weight gain may limit its clinical application for liver fibrosis.

As reviewed above, PPARs can not only improve lipid metabolism and insulin sensitivity but can also reduce liver inflammation. In the phase II trial of 276 patients with NASH without fibrosis treated with elafibranor (PPAR $\alpha / \delta$ agonist) for 1 year, the degree of fibrosis did not progress after receiving elafibranor $(120 \mathrm{mg} / \mathrm{d})$ versus the placebo group (19\% vs. $12 \% ; p=0.045) .{ }^{27}$ Although the reported elafibranor was well tolerated, renal impairment (increase in serum creatinine, $p<0.001$ ) or renal failure needs to be vigilantly monitored and further observed. The phase III trial of elafibranor for patients with NASH with fibrosis (F2- F3) is ongoing. We look forward to the further results of effectiveness and safety. In addition, a phase II trial (NCT03124108) of the efficacy of elafibranor in patients with primary biliary cholangitis has recently begun. These patients with primary biliary cholangitis are under-responsive to ursodeoxycholic acid. Whether this can reflect the antifibrotic effect is also awaited.

The other most promising categories of antifibrotics for NASH are FGF19 analog or FGF21 analog. FGF19 is a hormone that potently regulates CYP7A1-mediated bile acid homeostasis, inhibits fatty acid synthesis and increases fatty acid oxidation to maintain glucose homeostasis. Aldafermin (formerly NGM282), a FGF19 analog, treatment induced histological improvement in patients with NASH who received subcutaneous $3 \mathrm{mg}$ of aldafermin once daily for 12 weeks $(-0.5$ fibrosis score, $p=0.035)$. Significant reductions in fibrosis scores and neoepitope-specific $\mathrm{N}$-terminal propeptide of type III collagen are evident in 12 weeks $(-22 \%$ and $-33 \%$ in the $1 \mathrm{mg}$ and $3 \mathrm{mg}$ groups, respectively). ${ }^{28}$ At least one adverse event (AE) occurred in $93 \%$ of patients in the phase II trial for the treatment of NASH. Injection site reactions $(34 \%)$, diarrhea $(33 \%)$, abdominal pain $(18 \%)$, and nausea $(17 \%)$ were the most common AEs. ${ }^{29}$ Some results were also found in patients with primary sclerosing cholangitis after treatment with NGM282. Enhanced liver fibrosis scores were improved in 12 weeks $(-0.29, p=0.028$, in the $1 \mathrm{mg}$ group; $-0.37, p=0.009$, in the $3 \mathrm{mg}$ group); however, gastrointestinal symptoms were more frequent in the phase II trial. ${ }^{30}$ Also, pegbelfermin, an FGF21 analog, can regulate energy metabolism. In a phase IIa trial, 75 patients with NASH with fibrosis stages $1-3$ were treated with pegbelfermin $10 \mathrm{mg}$ or $20 \mathrm{mg}$ once per day. Data from 16 weeks of treatment showed that pegbelfermin led to a decrease in liver stiffness $(-6.8 \%$, $p=0.0004$, in the $10 \mathrm{mg}$ group; $-5.2 \%, p=0.0008$, in the 20 mg group). However, some common AEs occurred ( $16 \%$ diarrhea, $14 \%$ nausea). ${ }^{31}$ Although these side effects are mild, further observation is needed in future trials.

Although liver injury caused by different etiologies determines the initial mode of the liver fibrosis response, the pathological mechanism of fibrosis caused by different injury factors in the late stage of fibrosis is relatively consistent, such as bridging fibers between portal vein regions and cirrhosis. ${ }^{32}$ In any case, removal of the causative factor, such as weight loss in NAFLD or suppression of viral replica- tion in hepatitis $B / C$, is the basic treatment strategy to stimulate regression or reverse fibrosis. It should be noted that fibrosis may continue to progress in some patients in whom we are able to control or cure the primary disease, ${ }^{17,19}$ although the control of the primary disease is very effective in the treatment of fibrosis. Therefore, the mechanism of liver fibrosis still needs to be studied, while other strategies for the treatment of liver fibrosis still need to be carried out.

\section{Control of the inflammation}

The inflammatory response activates a variety of inflammatory cells and releases inflammatory cytokines, which makes HSCs change from a static state to an activated and proliferative state. Furthermore, it can lead to the deposition of ECM, and at the same time, it can also cause the disorder of liver immune function and further aggravate the injury of hepatocytes. Therefore, the inhibition of inflammation and the immune response are also important links in the treatment of fibrosis. Targeting inflammatory mediators or inhibiting the infiltration of inflammatory monocytes can reduce the formation of fibrosis.

Chemokines released by stress hepatocytes, Kupffer cells, endothelial cells and HSCs can regulate the recruitment of inflammatory cells (monocytes, neutrophils, lymphocytes) in the liver. ${ }^{33}$ The chemokine receptor CC chemokine receptor 2 (CCR2) is one of the core drivers of hepatic inflammation and fibrosis. ${ }^{34,35}$ It has been observed that the degree of hepatic fibrosis can be inhibited by targeting CCR2 in patients with NASH. ${ }^{36}$ The chemokine receptor CCR5 also contributes to fibrosis. $^{35}$

Cenicriviroc, a dual inhibitor of CCR2/CCR5, has been tested in patients with fibrotic NASH, producing exciting results. In phase IIb trials (CENTAUR), a total of 126 patients with NASH with bridging fibrosis and/or NAS $\geq 5$ treated with cenicriviroc $150 \mathrm{mg}$ were observed to yield a reduction in fibrosis. Even though antifibrotic effects have been reported, the safety of cenicriviroc should be considered carefully, as $2.8 \%$ of patients experienced fatigue and $2.1 \%$ of patients experienced diarrhea in 289 patients at year $1 .^{37}$ The antifibrotic effect of cenicriviroc was also shown in the final data at year 2.38 In addition, a phase III trial (AURORA, NCT03028740) is ongoing, and reports of the side effects of cenicriviroc are worthy of continuous follow-up. In the same way, cenicriviroc can also inhibit inflammation and reduce fibrosis by inhibiting hepatocyte death, ${ }^{39}$ balancing metabolism, ${ }^{40}$ or regulating the "gut-liver axis", ${ }^{41}$ which are also promising treatment strategies.

Other drugs aimed at blocking the recruitment of inflammatory cells such as macrophages, antioxidants, and hepatoprotectants are also in full-swing preclinical trials and may enter clinical development in the near future.

\section{Inhibition of cellular signaling pathways and cytokines to interfere with or block the activation of HSCS}

Many experiments have demonstrated the biological efficacy of fibrogenic cytokines that act in an autocrine or paracrine manner. In particular, TGF- $\beta$ is a master profibrogenic cytokine. The TGF- $\beta$ proteins comprise 3 isoforms: TGF- $\beta 1,2$, and 3. Mechanistically, TGF- $\beta 1$ is the predominant isoform in the pathogenesis of liver fibrosis. ${ }^{42}$ With activated canonical TGF$\beta$ signaling, targeted HSCs are transdifferentiated to MFs, inducing ECM production. In fact, inhibiting the 
overexpression and activity of TGF- $\beta$ has become a promising target of antifibrosis therapy. ${ }^{43,44}$ However, inhibition of TGF$\beta$ almost acts ubiquitously in all organisms, which may induce inflammation or tumors. Thus, limiting TGF- $\beta$ to directed fibrotic organs has become a challenge. ${ }^{45}$ These emerging cellular and signaling pathway mechanisms of liver fibrosis or cirrhosis provide the basis for research on antifibrotic strategies.

In addition, TGF- $\beta$ 's ligand-receptor binding and its signal transduction pathway may become potential targets for antifibrosis therapy. Research on such is in full-swing, and many experiments and clinical trials have already demonstrated that fibrosis can be slowed or reversed by inhibiting the activation of HSCs and regulating the signal-related pathway. Among them, cannabinoid receptor 1 (CB1) antagonist, angiotensin-converting enzyme inhibitor or angiotensin II receptor 1 blocker, endothelin 1 receptor antagonist, tyrosine kinase inhibitor, FXR antagonist, PPAR agonists, vitamin $D$ receptor, ${ }^{46}$ and adiponectin have shown potential for antifibrotic therapies. In particular, OCA (an FXR agonist) has demonstrated clinical benefit among patients with NASH in phase III clinical trial, ${ }^{47}$ pioglitazone (a PPAR $\gamma$ antagonist), ${ }^{48}$ rimonabant (CB1 antagonist) and other drugs are also undergoing trials. 49

OCA, as described above, might be the most promising drug candidate that reduces fibrosis in patients with NAFLD. Although treatment of patients with NASH with OCA given orally at $25 \mathrm{mg}$ daily for 72 weeks was found to be safe in a phase II (FLINT) trial; of note, $33(23 \%)$ of 141 patients in phase II developed pruritus. Moreover, pruritus in the REGENERATE trial (phase III, NCT02548351) in 1968 patients with NASH with stage F1-F3 fibrosis accumulated $(p=0.0002)$. Moderate to severe pruritus occurred in $336(51 \%)$ patients in the OCA $25 \mathrm{mg}$ group. ${ }^{47}$ Other AEs caused by OCA are elevated total cholesterol or low-density lipoprotein. The study of the efficacy and safety of OCA in patients with NASH with compensated cirrhosis in the phase III trial (REVERSE, NCT03439254) is ongoing. Thus, long-term efficacy and safety treatment with OCA need to be further considered.

Although studies of fibrosis pay close attention to intrahepatic cells and signaling pathways, it is important to realize that hepatic fibrosis is also greatly affected by extrahepatic events, including signals from the gut, fat, and muscles. All targeted therapies are effective in preclinical studies. The reason may be that the target is clear, but the actual clinical requirements for drug side effects are also very high and there will be a compensatory mechanism when a single target is blocked. Therefore, a very effective target drug has not been found and commercialized as antifibrotic therapy. In the future, in addition to further intervention with effective targets, combination therapy may also be a possible direction.

\section{Clearance of activated HSCs}

Promoting the apoptosis of activated HSCs, deactivation or direct reduction in the number of MFs may prevent the progression of liver fibrosis. The increased expression of

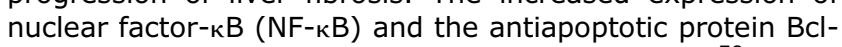
2 can lead to the continuous activation of HSCs. ${ }^{50}$ Druginduced apoptosis of HSCs by inhibiting NF-кB, including fraxetin (7,8-dihydroxy-6-methoxy coumarin $)^{51}$ and 4-hydroxy-2 $(3 \mathrm{H})$-benzoxazolone, ${ }^{52}$ has been identified in many animal experiments. Although these drugs may be potential antifibrotic agents, clinical trials have not yet begun. Therefore, it may be a long time before it can be used in the clinic. The clearance of HSCs or MFs by apoptosis can be controlled therapeutically. Recently, a novel molecular therapy that modulates $\mathrm{Bcl}-\mathrm{x}$ alternative splicing by an antisense oligonucleotide to induce HSC apoptosis may become a potential antifibrosis treatment strategy. ${ }^{53}$

In research, apoptosis signal-regulating kinase 1 (ASK1) can regulate the key apoptosis pathway of HSCs and hepatocytes, as well as the inflammatory signal. ${ }^{54}$ Selonsertib can reduce the activation of HSCs, collagen production, activation of inflammatory cytokine pathways and oxidative stress by inhibiting ASK-1. ${ }^{55}$ There is heartening evidence that 24 weeks treatment with selonsertib (6 or $18 \mathrm{mg}$, orally once daily) leads to improvement in fibrosis in patients with NASH with stage 2 or 3 fibrosis in a phase II trial. The proportion of patients with $a \geq 1$ stage improvement in fibrosis was $43 \%$ (13/30 patients) in the $18 \mathrm{mg}$ selonsertib group and $30 \%$ (8/27 patients) in the $6 \mathrm{mg}$ selonsertib group. ${ }^{54}$ Worryingly, many patients experienced at least one or more AEs. The most common AEs were nausea, headache, nasopharyngitis, upper abdominal pain, sinusitis, back pain, and fatigue. In fact, $6.9 \%$ of patients experienced serious AEs $(5 / 72)$, and $4.2 \%$ of patients discontinued treatment because of AEs (3/72). ${ }^{54}$ However, the phase III study of selonsertib (STELLAR-3 and STELLAR-4) did not meet the primary endpoint that fibrosis stage improvement without the progression of NASH. Selonsertib also did not reduce fibrosis in NASH patients with bridging fibrosis (F3) or compensated cirrhosis (F4) versus placebo at week 48. In STELLAR-3, the primary endpoint was achieved in $12 \%(p=0.93)$ of NASH patients with F3 in the selonsertib $6 \mathrm{mg}$ group and $10 \%$ of patients $(p=0.49)$ in the selonsertib $18 \mathrm{mg}$ group. In STELLAR-4, the proportion was $14 \%(p=0.56)$ and $13 \%$ $(p=0.93)$ in patients with $\mathrm{F} 4$, respectively. ${ }^{56}$ The present data showed no effect on reversing advanced fibrosis, while the serious AEs may also not be conducive to the promotion of drugs.

As an important part of innate immunity, natural killer (NK) cells can kill activated HSCs to enhance the immune surveillance ability of NK cells and activate their scavenging and killing effects, which could be an approach to scavenge activated HSCs. ${ }^{57}$ Therefore, the expansion of NK cells may be a new method for the treatment of liver fibrosis. However, hyperactivated NK cells can also lead to the progression of fibrosis by enhancing inflammation in the liver. ${ }^{58}$ Thus, understanding the balance of NK cells in regulating HSCs in patients with chronic liver disease can help us design novel antifibrotic therapies. The production of interferon- $\gamma$ is a marker of NK cell activation and a potent antifibrogenic cytokine contributing to inhibiting fibrogenesis via NK cells. Although the systemic use of interferon- $\gamma$ has no positive results and interferon- $\gamma$-related side effects are inevitable, engineered targeted interferon- $\gamma$ offers new hope as it can inhibit the activation of HSCs in carbon tetrachloride-induced fibrosis in a mouse model but does not induce related side effects. ${ }^{59}$

\section{Inhibition of the production of ECM and promotion of degradation}

Emerging antifibrosis therapy aims to inhibit the production of ECM and/or prevent the deposition of ECM protein. ECM is a critical determinant of cell and tissue function in fibrosis. 
Matrix metalloproteinases (MMPs) are a family of more than 24 zinc-dependent endopeptidases that can degrade any component of the ECM. ${ }^{60}$ According to their ECM substrate specificity, MMPs have been divided into five categories: gelatinases, collagenases, membrane-type, matrilysins, and stromelysins. ${ }^{61}$ MMPs can not only degrade ECM proteins but also act on non-ECM substrates, such as chemokines and cytokines, which can modulate cell inflammation. ${ }^{62}$ In the liver fibrosis rat model, carbon tetrachloride and bile duct ligation confirmed that suppressed tissue inhibitor of metalloproteinase (TIMP)-1 expression can inhibit the formation of liver fibrosis by promoting ECM degradation. ${ }^{63}$

TIMP is a family of at least four physiological inhibitors (TIMPs 1-4) that can regulate proteolytic activity in tissues. Chronic inflammation and repeated repair processes lead to excessive accumulation of ECM components, such as collagen, fibronectin and proteoglycans, which are major participants in the formation of scar tissue. Both MMPs and TIMPs are considered to play central roles in the development of liver fibrosis at different time periods. Basic studies have shown that the balance between MMPs and TIMPs plays an important role in the homeostasis of ECM content. In addition, the expression and activity of MMPs and TIMPs are necessary to ensure fibrinolysis during the regression of fibrosis. These are expected to become therapeutic targets for new drugs.

Similarly, lysyl oxidase-like protein 2 (LOXL2) can promote the cross-linking and stabilization of type I collagen, which is the key to the progression or regeneration of fibrosis. Some experiments have shown that it is effective to use the inhibitory monoclonal LOXL2 antibody AB0023 for early treatment in a mouse model of mild liver fibrosis. ${ }^{64}$ Although these studies have shown that targeted LOXL2 inhibition is one of the treatments for the prevention or regression of liver fibrosis, it still needs to be tested in clinical trials. Simtuzumab (formerly GS-6624), a monoclonal antibody directed against the LOXL2 enzyme produced by Gilead Sciences, has completed a clinical trial in human immunodeficiency virus- and/ or HCV-infected adults with liver fibrosis. However, there was no significant improvement the Ishak fibrosis stage after simtuzumab treatment for 96 weeks ( $p=0.12 \mathrm{vs}$. placebo, in the $75 \mathrm{mg} \mathrm{Arm} ; p=0.13$ vs. placebo, in the $125 \mathrm{mg} \mathrm{Arm}){ }^{65}$

Studies have shown that pirfenidone can effectively reduce the expression of heat shock protein-47 (HSP-47) and reduce the abnormal accumulation of collagen I and collagen III and down-regulate the expression of collagen II, TIMP-1 and MMP2 by regulating the activity of the TGF- $\beta$ signaling pathway, effectively reducing collagen deposition by $70 \%$, inhibiting HSC proliferation and serum transaminase levels, and preventing balloon degeneration of hepatocytes. Pirfenidone treatment reduces liver inflammation and fibrosis in patients with $\mathrm{HCV}$. The Ishak fibrosis stage improved two points in $67 \%(p<0.05)$ of patients with chronic hepatitis C after receipt of study drugs at the 24 -month point. ${ }^{66}$ Despite the encouraging results, there are still concerns about the potential AEs associated with pirfenidone. ${ }^{67}$ In reports on the treatment of pulmonary fibrosis with pirfenidone, the median time to develop an AE after the use of pirfenidone was 15 days. ${ }^{68}$ Gastrointestinal symptoms (nausea, vomiting, and diarrhea) and skin-related AEs (rash and photosensitivity) are the most common AEs caused by pirfenidone. Liver function AEs and fatigue associated with the treatment of pirfenidone also need to be monitored. ${ }^{69}$ Thus, further clinical trials are needed to confirm the safety in patients with fibrosis. But more worrying is that pirfenidone exhibited less of an antifibrotic effect in advanced liver fibrosis. ${ }^{70,71}$

HSP-47 plays a conclusive role in the secretion and maturation of collagen and other ECM. BMS-986263 is a targeted lipid nanoparticle delivering HSP-47 small interference RNA. Recently, the efficacy of BMS-986263 was announced at the 2019 meeting of the American Association for the Study of Liver Diseases. Although with a limited number of participants in this trial, the Ishak fibrosis stage was improved in the patients with advanced fibrosis after cure of HCV at week 12 (NCT03420768).

It is critical to recognize that preventing the inhibition of ECM and promoting its degradation can help the treatment of patients with advanced fibrosis or cirrhosis. Continued experimental advances are flourishing, yet most of these studies have not been carried out in humans. Therefore, there may be a long way to go to develop effective antifibrotic drugs by halting the progression or inducing the regression of ECM proteins.

\section{Summary and perspective}

Pharmacotherapy for hepatic antifibrotic continues to represent major unmet medical needs. We have summarized the major targets for the most promising pharmacological agents in clinical trials in Fig. 3. Although with different shortcomings, a number of drugs have been investigated in phase III clinical trials and provide great hope for antifibrosis therapy in the future. The research and development of newly emerging pharmaceutics targeting different signaling pathways and targets will be helpful to reduce the burden of chronic liver disease and will reduce the number of hepatic decompensations or HCC. In addition, some traditional Chinese medicines, such as Fuzheng Huayu, ${ }^{72}$ Biejia Ruangan ${ }^{73}$ and Ganshuang granules, ${ }^{74}$ have gratifying antifibrosis effects in China. The effect of these traditional Chinese medicines on reducing liver fibrosis has also been confirmed. ${ }^{75}$ However, the treatment of advanced fibrosis and liver cirrhosis may still take longer to complete reversal, and drug research for patients with irreversible liver cirrhosis is also a challenge.

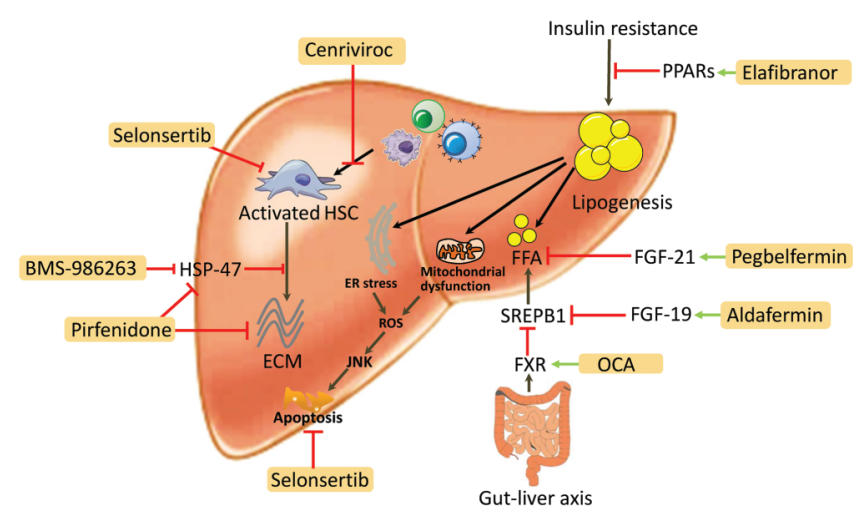

Fig. 3. Major targets for the most promising pharmacological agents in clinical trials.

Abbreviations: CCR, CC chemokine receptor; ECM, extracellular matrix; ER, endoplasmic reticulum; FGF, fibroblast growth factor; FFA, free fatty acid; FXR, farnesoid $X$ receptor; $H S C$, hepatic stellate cell; HSP, heat shock protein; JNK, c-Jun N-terminal kinase; PPARs, peroxisome proliferator-activated receptor; ROS, reactive oxygen species; SREPB1, sterol regulatory element binding protein 1. 
The emergence and development of fibrosis is a multifactor, multistep complex process, so it may be difficult to make a breakthrough in the treatment of a single target, a pathway, or a single link. Thus, it is an ideal option to develop a combination therapeutic strategy on multiple pathways. The combination of drugs should involve therapy strategies for curing or controlling the primary disease along with direct as well as indirect antifibrotic approaches. Anyhow, it can be expected that research on antifibrotic drugs will continue to be popular for a long time in the future. We will hopefully witness the success of the strategy of hepatic antifibrotic therapy for further improving the effectiveness and safety of treatment to improve outcomes in the near future.

\section{Funding}

This work was supported by the Tianjin Science and Technology Project (15ZXLCSY00040).

\section{Conflict of interest}

The authors have no conflict of interests related to this publication.

\section{Author contributions}

Review of the literature and drafting of the manuscript (YC), critical revision of the manuscript $(\mathrm{HL})$.

\section{References}

[1] Schuppan D. Liver fibrosis: Common mechanisms and antifibrotic therapies. Clin Res Hepatol Gastroenterol 2015;39 Suppl 1:S51-S59. doi: 10.1016/j. clinre.2015.05.005.

[2] Nishio T, Hu R, Koyama Y, Liang S, Rosenthal SB, Yamamoto G, et al. Activated hepatic stellate cells and portal fibroblasts contribute to cholestatic liver fibrosis in MDR2 knockout mice. J Hepatol 2019;71:573-585. doi: 10. 1016/j.jhep.2019.04.012.

[3] Hempel F, Roderfeld M, Savai R, Sydykov A, Irungbam K, Schermuly R, et al. Depletion of bone marrow-derived fibrocytes attenuates TAA-induced liver fibrosis in mice. Cells 2019;8:1210. doi: 10.3390/cells8101210.

[4] Iwaisako K, Jiang C, Zhang M, Cong M, Moore-Morris TJ, Park TJ, et al. Origin of myofibroblasts in the fibrotic liver in mice. Proc Natl Acad Sci U S A 2014; 111:E3297-E3305. doi: 10.1073/pnas.1400062111.

[5] Chu AS, Diaz R, Hui JJ, Yanger K, Zong Y, Alpini G, et al. Lineage tracing demonstrates no evidence of cholangiocyte epithelial-to-mesenchymal transition in murine models of hepatic fibrosis. Hepatology 2011;53:1685-1695. doi: $10.1002 /$ hep. 24206 .

[6] Lepreux S, Desmoulière A. Human liver myofibroblasts during development and diseases with a focus on portal (myo)fibroblasts. Front Physiol 2015;6: 173. doi: $10.3389 /$ fphys.2015.00173.

[7] Huang $\mathrm{YH}$, Chen $\mathrm{MH}$, Guo $\mathrm{OL}$, Chen $\mathrm{ZX}$, Chen $\mathrm{OD}$, Wang $X Z$. Interleukin-10 induces senescence of activated hepatic stellate cells via STAT3-p53 pathway to attenuate liver fibrosis. Cell Signal 2020;66:109445. doi: 10.1016/j. cellsig.2019.109445.

[8] Yu HX, Yao Y, Bu FT, Chen Y, Wu YT, Yang Y, et al. Blockade of YAP alleviates hepatic fibrosis through accelerating apoptosis and reversion of activated hepatic stellate cells. Mol Immunol 2019;107:29-40. doi: 10.1016/j. molimm.2019.01.004

[9] Krizhanovsky V, Yon M, Dickins RA, Hearn S, Simon J, Miething C, et al. Senescence of activated stellate cells limits liver fibrosis. Cell 2008;134: 657-667. doi: 10.1016/j.cell.2008.06.049.

[10] Tsuchida T, Friedman SL. Mechanisms of hepatic stellate cell activation. Nat Rev Gastroenterol Hepatol 2017;14:397-411. doi: 10.1038/nrgastro.2017.38.

[11] Li H, You H, Fan X, Jia J. Hepatic macrophages in liver fibrosis: pathogenesis and potential therapeutic targets. BMJ Open Gastroenterol 2016;3:e000079. doi: 10.1136/bmjgast-2016-000079.

[12] Hernández-Gea V, Ghiassi-Nejad Z, Rozenfeld R, Gordon R, Fiel MI, Yue Z, et al. Autophagy releases lipid that promotes fibrogenesis by activated hepatic stellate cells in mice and in human tissues. Gastroenterology 2012;142:938-946. doi: 10.1053/j.gastro.2011.12.044
[13] Coll M, El Taghdouini A, Perea L, Mannaerts I, Vila-Casadesús M, Blaya D, et al. Integrative miRNA and Gene Expression Profiling Analysis of Human Quiescent Hepatic Stellate Cells. Sci Rep 2015;5:11549. doi: 10. 1038 /srep 11549.

[14] Wang P, Lei S, Wang X, Xu W, Hu P, Chen F, et al. MicroRNA-134 deactivates hepatic stellate cells by targeting TGF- $\beta$ activated kinase 1 -binding protein 1 . Biochem Cell Biol 2019;97:505-512. doi: 10.1139/bcb-2018-0211.

[15] Papatheodoridis GV, Idilman R, Dalekos GN, Buti M, Chi H, van Boemmel F, et al. The risk of hepatocellular carcinoma decreases after the first 5 years of entecavir or tenofovir in Caucasians with chronic hepatitis B. Hepatology 2017;66:1444-1453. doi: 10.1002/hep.29320.

[16] Kim SU, Seo YS, Lee HA, Kim MN, Lee YR, Lee HW, et al. A multicenter study of entecavir vs. tenofovir on prognosis of treatment-naïve chronic hepatitis B in South Korea. J Hepatol 2019;71:456-464. doi: 10.1016/j. jhep.2019.03.028.

[17] D'Ambrosio R, Aghemo A, Rumi MG, Ronchi G, Donato MF, Paradis V, et al. A morphometric and immunohistochemical study to assess the benefit of a sustained virological response in hepatitis $C$ virus patients with cirrhosis. Hepatology 2012;56:532-543. doi: 10.1002/hep.25606.

[18] Glass LM, Dickson RC, Anderson JC, Suriawinata AA, Putra J, Berk BS, et al. Total body weight loss of $\geq 10 \%$ is associated with improved hepatic fibrosis in patients with nonalcoholic steatohepatitis. Dig Dis Sci 2015;60:10241030. doi: 10.1007/s10620-014-3380-3.

[19] Maylin S, Martinot-Peignoux M, Moucari R, Boyer N, Ripault MP, CazalsHatem $D$, et al. Eradication of hepatitis $C$ virus in patients successfully treated for chronic hepatitis C. Gastroenterology 2008;135:821-829. doi: 10.1053/j.gastro.2008.05.044.

[20] Poynard T, Moussalli J, Munteanu M, Thabut D, Lebray P, Rudler M, et al. Slow regression of liver fibrosis presumed by repeated biomarkers after virological cure in patients with chronic hepatitis C. J Hepatol 2013;59:675-683. doi: 10.1016/j.jhep.2013.05.015.

[21] Anstee QM, Reeves HL, Kotsiliti E, Govaere O, Heikenwalder M. From NASH to HCC: current concepts and future challenges. Nat Rev Gastroenterol Hepatol 2019;16:411-428. doi: 10.1038/s41575-019-0145-7.

[22] Arab JP, Arrese M, Trauner M. Recent Insights into the Pathogenesis of Nonalcoholic Fatty Liver Disease. Annu Rev Pathol 2018;13:321-350. doi: 10. 1146/annurev-pathol-020117-043617.

[23] Molinaro A, Wahlström A, Marschall HU. Role of Bile Acids in Metabolic Control. Trends Endocrinol Metab 2018;29:31-41. doi: 10.1016/j.tem. 2017.11.002.

[24] Mudaliar S, Henry RR, Sanyal AJ, Morrow L, Marschall HU, Kipnes M, et al. Efficacy and safety of the farnesoid $X$ receptor agonist obeticholic acid in patients with type 2 diabetes and nonalcoholic fatty liver disease. Gastroenterology 2013;145:574-582.e1. doi: 10.1053/j.gastro.2013.05.042.

[25] Neuschwander-Tetri BA, Loomba R, Sanyal AJ, Lavine JE, Van Natta ML, Abdelmalek MF, et al. Farnesoid $X$ nuclear receptor ligand obeticholic acid for non-cirrhotic, non-alcoholic steatohepatitis (FLINT): a multicentre, randomised, placebo-controlled trial. Lancet 2015;385:956-965. doi: 10. 1016/S0140-6736(14)61933-4.

[26] Bril F, Kalavalapalli S, Clark VC, Lomonaco R, Soldevila-Pico C, Liu IC, et al. Response to pioglitazone in patients with nonalcoholic steatohepatitis with vs without type 2 diabetes. Clin Gastroenterol Hepatol 2018;16:558-566.e2. doi: $10.1016 /$ j.cgh.2017.12.001.

[27] Ratziu V, Harrison SA, Francque S, Bedossa $P$, Lehert $P$, Serfaty $L$, et al. Elafibranor, an agonist of the peroxisome proliferator-activated receptor- $\alpha$ and $-\delta$, induces resolution of nonalcoholic steatohepatitis without fibrosis worsening. Gastroenterology 2016;150:1147-1159.e5. doi: 10.1053/j. gastro.2016.01.038

[28] Harrison SA, Rossi SJ, Paredes AH, Trotter JF, Bashir MR, Guy CD, et al. NGM282 improves liver fibrosis and histology in 12 weeks in patients with nonalcoholic steatohepatitis. Hepatology 2020;71:1198-1212. doi: 10. 1002/hep. 30590

[29] Harrison SA, Rinella ME, Abdelmalek MF, Trotter JF, Paredes AH, Arnold HL, et al. NGM282 for treatment of non-alcoholic steatohepatitis: a multicentre, randomised, double-blind, placebo-controlled, phase 2 trial. Lancet 2018 391:1174-1185. doi: 10.1016/S0140-6736(18)30474-4.

[30] Hirschfield GM, Chazouillères O, Drenth JP, Thorburn D, Harrison SA, Landis CS, et al. Effect of NGM282, an FGF19 analogue, in primary sclerosing cholangitis: A multicenter, randomized, double-blind, placebo-controlled phase II trial. J Hepatol 2019;70:483-493. doi: 10.1016/j.jhep.2018.10.035.

[31] Sanyal A, Charles ED, Neuschwander-Tetri BA, Loomba R, Harrison SA, Abdelmalek MF, et al. Pegbelfermin (BMS-986036), a PEGylated fibroblast growth factor 21 analogue, in patients with non-alcoholic steatohepatitis: a randomised, double-blind, placebo-controlled, phase 2a trial. Lancet 2019; 392:2705-2717. doi: 10.1016/S0140-6736(18)31785-9.

[32] Tapper EB, Lok AS. Use of liver imaging and biopsy in clinical practice. N Engl J Med 2017;377:756-768. doi: 10.1056/NEJMra1610570.

[33] Marra F, Tacke F. Roles for chemokines in liver disease. Gastroenterology 2014;147:577-594.e1. doi: 10.1053/j.gastro.2014.06.043.. 
[34] Parker R, Weston CJ, Miao Z, Corbett C, Armstrong MJ, Ertl L, et al. CC chemokine receptor 2 promotes recruitment of myeloid cells associated with insulin resistance in nonalcoholic fatty liver disease. Am J Physio Gastrointest Liver Physiol 2018;314:G483-G493. doi: 10.1152/ajpgi. 00213.2017

[35] Fantuzzi L, Tagliamonte M, Gauzzi MC, Lopalco L. Dual CCR5/CCR2 targeting: opportunities for the cure of complex disorders. Cell Mol Life Sci 2019;76: 4869-4886. doi: 10.1007/s00018-019-03255-6.

[36] Krenkel O, Puengel T, Govaere O, Abdallah AT, Mossanen JC, Kohlhepp M, et al. Therapeutic inhibition of inflammatory monocyte recruitment reduces steatohepatitis and liver fibrosis. Hepatology 2018;67:1270-1283. doi: 10 . 1002/hep.29544.

[37] Friedman S, Sanyal A, Goodman Z, Lefebvre E, Gottwald M, Fischer L, et al. Efficacy and safety study of cenicriviroc for the treatment of non-alcoholic steatohepatitis in adult subjects with liver fibrosis: CENTAUR Phase $2 \mathrm{~b}$ study design. Contemp Clin Trials 2016;47:356-365. doi: 10.1016/j.cct. 2016.02.012.

[38] Ratziu V, Sanyal A, Harrison SA, Wong VW, Francque S, Goodman Z, et al. Cenicriviroc treatment for adults with nonalcoholic steatohepatitis and fibrosis: Final analysis of the phase 2b CENTAUR Study. Hepatology 2020. doi: 10.1002/hep.31108.

[39] Wree A, Mehal WZ, Feldstein AE. Targeting cell death and sterile inflammation loop for the treatment of nonalcoholic steatohepatitis. Semin Liver Dis 2016;36:27-36. doi: 10.1055/s-0035-1571272.

[40] Sumida Y, Yoneda M, Tokushige K, Kawanaka M, Fujii H, Yoneda M, et al. Antidiabetic therapy in the treatment of nonalcoholic steatohepatitis. Int J Mol Sci 2020;21:1907. doi: 10.3390/ijms21061907.

[41] Woodhouse CA, Patel VC, Singanayagam A, Shawcross DL. Review article: the gut microbiome as a therapeutic target in the pathogenesis and treatment of chronic liver disease. Aliment Pharmacol Ther 2018;47:192-202. doi: 10.1111/apt.14397.

[42] Fabregat I, Moreno-Càceres J, Sánchez A, Dooley S, Dewidar B, Giannelli G, et al. TGF- $\beta$ signalling and liver disease. FEBS J 2016;283:2219-2232. doi: 10.1111 /febs. 13665 .

[43] Abd El-Meguid M, Dawood RM, Mokhles MA, El Awady MK. Extrahepatic upregulation of transforming growth factor beta 2 in HCV genotype 4-induced liver fibrosis. J Interferon Cytokine Res 2018;38:341-347. doi: 10. 1089/jir.2018.0045.

[44] Fan W, Liu T, Chen W, Hammad S, Longerich T, Hausser I, et al. ECM1 prevents activation of transforming growth factor $\beta$, hepatic stellate cells, and fibrogenesis in mice. Gastroenterology 2019;157:1352-1367.e1313. doi: 10.1053/j.gastro.2019.07.036.

[45] Dewidar B, Meyer C, Dooley S, Meindl-Beinker AN. TGF- $\beta$ in hepatic stellate cell activation and liver fibrogenesis-updated 2019. Cells 2019;8:1419. doi: $10.3390 /$ cells 8111419 .

[46] Udomsinprasert W, Jittikoon J. Vitamin D and liver fibrosis: Molecular mechanisms and clinical studies. Biomed Pharmacother 2019;109:1351-1360. doi: 10.1016/j.biopha.2018.10.140.

[47] Younossi ZM, Ratziu V, Loomba R, Rinella M, Anstee QM, Goodman Z, et al. Obeticholic acid for the treatment of non-alcoholic steatohepatitis: interim analysis from a multicentre, randomised, placebo-controlled phase 3 trial. Lancet 2019;394:2184-2196. doi: 10.1016/S0140-6736(19)33041-7.

[48] Musso G, Cassader M, Paschetta E, Gambino R. Thiazolidinediones and advanced liver fibrosis in nonalcoholic steatohepatitis: A meta-analysis. JAMA Intern Med 2017;177:633-640. doi: 10.1001/jamainternmed.2016. 9607.

[49] Issa YA, El Achy SN, Mady RF. Cannabinoid receptor-1 antagonism: a new perspective on treating a murine schistosomal liver fibrosis model. Mem Inst Oswaldo Cruz 2019;114:e190062. doi: 10.1590/0074-02760190062.

[50] Watson MR, Wallace K, Gieling RG, Manas DM, Jaffray E, Hay RT, et al. NF-kappaB is a critical regulator of the survival of rodent and human hepatic myofibroblasts. J Hepatol 2008;48:589-597. doi: 10.1016/j.jhep. 2007.12.019.

[51] Wu B, Wang R, Li S, Wang Y, Song F, Gu Y, Yuan Y. Antifibrotic effects of Fraxetin on carbon tetrachloride-induced liver fibrosis by targeting NF$\kappa \mathrm{B} / \mathrm{I} \kappa \mathrm{B} \alpha, \mathrm{MAPKs}$ and $\mathrm{Bcl}-2 / \mathrm{Bax}$ pathways. Pharmacol Rep 2019;71:409416. doi: 10.1016/j.pharep.2019.01.008.

[52] Sun X, Huang X, Zhu X, Liu L, Mo S, Wang $\mathrm{H}$, et al. HBOA ameliorates $\mathrm{CCl}_{4}$ incuded liver fibrosis through inhibiting TGF- $\beta 1 /$ Smads, NF-кB and ERK signaling pathways. Biomed Pharmacother 2019;115:108901. doi: 10.1016/j. biopha.2019.108901.

[53] Wu L, Mao C, Ming X. Modulation of Bcl-x alternative splicing induces apoptosis of human hepatic stellate cells. Biomed Res Int 2016;2016:7478650. doi: $10.1155 / 2016 / 7478650$.

[54] Loomba R, Lawitz E, Mantry PS, Jayakumar S, Caldwell SH, Arnold H, et al. The ASK1 inhibitor selonsertib in patients with nonalcoholic steatohepatitis: A randomized, phase 2 trial. Hepatology 2018;67:549-559. doi: 10 1002/hep.29514.
[55] Xiang $M$, Wang PX, Wang AB, Zhang XJ, Zhang $Y$, Zhang $P$, et al. Targeting hepatic TRAF1-ASK1 signaling to improve inflammation, insulin resistance, and hepatic steatosis. J Hepatol 2016;64:1365-1377. doi: 10.1016/j.jhep. 2016.02.002.

[56] Harrison SA, Wong VW, Okanoue T, Bzowej N, Vuppalanchi R, Younes Z, et al. Selonsertib for patients with bridging fibrosis or compensated cirrhosis due to NASH: Results from randomized phase III STELLAR trials. J Hepatol 2020. doi: $10.1016 / j$.jhep.2020.02.027.

[57] Wang $\mathrm{H}$, Yin S. Natural killer T cells in liver injury, inflammation and cancer. Expert Rev Gastroenterol Hepatol 2015;9:1077-1085. doi: 10. 1586/17474124.2015.1056738.

[58] Wei X, Qian J, Yao W, Chen L, Guan H, Chen Y, et al. Hyperactivated peripheral invariant natural killer T cells correlate with the progression of HBV-relative liver cirrhosis. Scand J Immunol 2019;90:e12775. doi: 10.1111/sji. 12775.

[59] Bansal R, Prakash J, Post E, Beljaars L, Schuppan D, Poelstra K. Novel engineered targeted interferon-gamma blocks hepatic fibrogenesis in mice. Hepatology 2011;54:586-596. doi: 10.1002/hep.24395.

[60] Roderfeld M. Matrix metalloproteinase functions in hepatic injury and fibrosis. Matrix Biol 2018;68-69:452-462. doi: 10.1016/j.matbio.2017.11.011.

[61] Kessenbrock K, Plaks V, Werb Z. Matrix metalloproteinases: regulators of the tumor microenvironment. Cell 2010;141:52-67. doi: 10.1016/j.cell.2010. 03.015.

[62] Cui N, Hu M, Khalil RA. Biochemical and biological attributes of matrix metalloproteinases. Prog Mol Biol Transl Sci 2017;147:1-73. doi: 10.1016/bs. pmbts.2017.02.005.

[63] Cong M, Liu T, Wang P, Fan X, Yang A, Bai $Y$, et al. Antifibrotic effects of a recombinant adeno-associated virus carrying small interfering RNA targeting TIMP-1 in rat liver fibrosis. Am J Pathol 2013;182:1607-1616. doi: 10. 1016/j.ajpath.2013.01.036.

[64] Ikenaga N, Peng ZW, Vaid KA, Liu SB, Yoshida S, Sverdlov DY, et al. Selective targeting of lysyl oxidase-like 2 (LOXL2) suppresses hepatic fibrosis progression and accelerates its reversal. Gut 2017;66:1697-1708. doi: 10 . 1136/gutjnl-2016-312473.

[65] Meissner EG, McLaughlin M, Matthews L, Gharib AM, Wood BJ, Levy E, et al. Simtuzumab treatment of advanced liver fibrosis in HIV and HCV-infected adults: results of a 6-month open-label safety trial. Liver Int 2016;36:17831792. doi: 10.1111/liv.13177.

[66] Flores-Contreras L, Sandoval-Rodríguez AS, Mena-Enriquez MG, LucanoLanderos S, Arellano-Olivera I, Alvarez-Álvarez A, et al. Treatment with pirfenidone for two years decreases fibrosis, cytokine levels and enhances CB2 gene expression in patients with chronic hepatitis C. BMC Gastroenterol 2014;14:131. doi: 10.1186/1471-230X-14-131.

[67] Verma N, Kumar P, Mitra S, Taneja S, Dhooria S, Das A, et al. Drug idiosyncrasy due to pirfenidone presenting as acute liver failure: Case report and mini-review of the literature. Hepatol Commun 2017;2:142-147. doi: 10. 1002/hep4.1133.

[68] Lancaster LH, de Andrade JA, Zibrak JD, Padilla ML, Albera C, Nathan SD, et al. Pirfenidone safety and adverse event management in idiopathic pulmonary fibrosis. Eur Respir Rev 2017;26:170057. doi: 10.1183/16000617. 0057-2017.

[69] Kropski JA, Blackwell TS. Progress in understanding and treating idiopathic pulmonary fibrosis. Annu Rev Med 2019;70:211-224. doi: 10 . 1146/annurev-med-041317-102715.

[70] Xiang XH, Jiang TP, Zhang S, Song J, Li X, Yang JY, et al. Pirfenidone inhibits proliferation, arrests the cell cycle, and downregulates heat shock protein-47 and collagen type I in rat hepatic stellate cells in vitro. Mol Med Rep 2015;12: 309-314. doi: 10.3892/mmr.2015.3403.

[71] Seniutkin O, Furuya S, Luo YS, Cichocki JA, Fukushima H, Kato $Y$, et al. Effects of pirfenidone in acute and sub-chronic liver fibrosis, and an initiation-promotion cancer model in the mouse. Toxicol Appl Pharmacol 2018; 339:1-9. doi: 10.1016/j.taap.2017.11.024.

[72] Chen J, Hu Y, Chen L, Liu W, Mu Y, Liu P. The effect and mechanisms of Fuzheng Huayu formula against chronic liver diseases. Biomed Pharmacother 2019;114:108846. doi: 10.1016/j.biopha.2019.108846.

[73] Huang $C$, Shen $D$, Sun $S$, Huang $Y$, Xin $Y$, Luo $H$, et al. Effect of Fufang Biejia Ruangan Tablet on lowering biochemical and virological parameters of hepatic fibrosis in patients with chronic hepatitis B: Protocol for a systematic review and meta-analysis of randomized controlled trials and cohort studies. Medicine (Baltimore) 2019;98:e15297. doi: 10.1097/MD. 0000000000015297.

[74] Shi H, Shi H, Ren F, Chen D, Chen Y, Duan Z. Naringin in Ganshuang Granule suppresses activation of hepatic stellate cells for anti-fibrosis effect by inhibition of mammalian target of rapamycin. J Cell Mol Med 2017;21:500-509. doi: $10.1111 /$ jcmm.12994.

[75] Li H. Advances in anti hepatic fibrotic therapy with Traditional Chinese Medicine herbal formula. J Ethnopharmacol 2020;251:112442. doi: 10.1016/j. jep.2019.112442. 